\title{
Mapping health-related quality of life scores from FACT-G, FAACT, and FACIT-F onto preference-based EQ-5D-5L utilities in non-small cell lung cancer cachexia
}

\author{
Michela Meregaglia $^{1,2} \cdot$ Ludovica Borsoi $^{1} \cdot$ John Cairns $^{2,4} \cdot$ Rosanna Tarricone $^{1,3}$
}

Received: 1 September 2016 / Accepted: 25 August 2017 / Published online: 25 September 2017

(C) The Author(s) 2017. This article is an open access publication

\begin{abstract}
Background Health-related quality of life (HRQoL) measurements from disease-specific tools cannot be directly used in economic evaluations. This study aimed to develop and validate mapping algorithms that predicted EuroQol 5-Dimensions 5-Levels (EQ-5D-5L) utilities from Functional Assessment of Anorexia-Cachexia Therapy (FAACT) and Functional Assessment of Chronic Illness TherapyFatigue (FACIT-F) and their common component (Functional Assessment of Cancer Therapy-General-FACT-G) in patients with non-small cell lung cancer cachexia.

Methods Data were collected on five occasions over a 12-week period in two multicenter placebo-controlled trials. EQ-5D-5L utilities were calculated using both English and Dutch value sets. The study sample was divided into development and validation datasets according to patients' geographical residence. Generalized estimating equations were applied to five different sets of independent variables including overall, Trial Outcome Index (TOI), and individual
\end{abstract}

Electronic supplementary material The online version of this article (doi:10.1007/s10198-017-0930-6) contains supplementary material, which is available to authorized users.

Michela Meregaglia

michela.meregaglia@unibocconi.it

1 CeRGAS (Research Centre on Health and Social Care Management), Bocconi University, Via Roentgen 1, 20136 Milan, Italy

2 Department of Health Services Research, London School of Hygiene and Tropical Medicine (LSHTM), London, UK

3 Department of Policy Analysis and Public Management, Bocconi University, Milan, Italy

4 CCBIO (Centre for Cancer Biomarkers), University of Bergen, Bergen, Norway subscales results. The best performing models were selected based on mean absolute error (MAE) and root-mean square error (RMSE).

Results EQ-5D-5L and FAACT/FACIT-F results were available for 96 patients. The developed algorithms showed a good predictive performance, with acceptable MAE/ RMSE and small differences between mean observed and predicted EQ-5D-5L utilities. In FACT-G models, Physical Well-Being had the highest explanatory value, while Emotional Well-Being did not significantly affect the EQ-5D-5L score; Anorexia-Cachexia and Fatigue subscales were highly statistically significant in FAACT and FACIT-F models, respectively, as well as the TOI scores. The Eastern Cooperative Oncology Group status was included as covariate in all models.

Conclusion The developed algorithms enable the estimation of EQ-5D-5L utilities from three cancer-specific instruments when preference-based HRQoL data are missing.

Keywords Mapping - FAACT - FACIT-F · FACT-G · EQ-5D-5L $\cdot$ Non-small cell lung cancer cachexia

JEL Classification $\quad \mathrm{C} 2 \cdot \mathrm{I} 1$

\section{Introduction}

Cachexia has been defined as "a complex metabolic syndrome associated with underlying illness and characterized by the loss of muscle with or without the loss of fat mass" [1]. Cachexia is a common clinical manifestation in cancer, especially at advanced stages. The frequency of this syndrome in lung cancer consistently outweighs that in other malignancies [2]. Cachectic patients experience a wide range of symptoms including lack of appetite, early satiety, and 
impaired physical functioning, which cumulatively decrease their quality of life and worsen their prognosis. Indeed, cachexia is estimated to be the direct cause of at least $20 \%$ of cancer deaths $[3,4]$.

The questionnaires belonging to the functional assessment of chronic illness therapy (FACIT) measurement system [5] are frequently adopted to estimate health-related quality of life (HRQoL) in cancer studies. The functional assessment of cancer therapy-general (FACT-G) can be used in any tumor type and constitutes the core of all other questionnaires addressing the specific concerns of patients with various malignancies. Among them, the functional assessment of anorexia/cachexia treatment (FAACT) has been recommended in the assessment of cancer-related cachexia [6]. The questionnaire may be administered in conjunction with others such as the functional assessment of chronic illness therapy-fatigue (FACIT-F), which is specifically aimed at measuring fatigue symptoms in chronic diseases. However, these questionnaires do not provide preference-based scores (utilities) that are essential in the quality-adjusted life years (QALYs) calculation for cost-effectiveness analyses. Conversely, the EuroQol five-dimensional (EQ-5D) questionnaire, developed by the group EuroQol [7], furnishes preference weights from the general population to derive QALYs and has been endorsed as a health utility standard by the National Institute for Health and Care Excellence (NICE) in England [8].

The measurement of HRQoL in oncology is mostly carried out using cancer-specific instruments rather than generic preference-based measures as they focus on relevant health issues and tend to capture more meaningful changes in symptomatology [9]. In a systematic literature review of studies addressing HRQoL in cancer anorexia-cachexia syndrome [10], FAACT was endorsed by seven studies, one of them reporting also the common core component (FACT-G), while only four studies adopted the generic EQ-5D instrument. In the absence of preference-based scores, statistical models that "map", or "cross-walk", the responses from a disease-specific instrument to utility values can be used as an alternative solution. In recent years, there has been a growing interest in mapping, with a number of published algorithms predicting EQ-5D health utilities from a wide range of disease-specific, non-preference-based scores [11]. A database maintained by the Health Economics Research Centre (Oxford University) [12] and regularly updated with all mapping studies published in the literature yielded 24 algorithms estimating EQ-5D scores from cancer-specific instruments; among them, eight studies adopted a questionnaire from the FACIT group to address a variety of tumors (breast, prostate, colorectal, lung, and melanoma). However, no algorithm exists specifically for anorexia-cachexia cancer syndrome, thus making it difficult for regulatory bodies to assess the QALY gain of new treatments for the purpose of reimbursement in the absence of generic preference-based data collections. The objective of this study was to develop a reliable mapping function to estimate the 5-level EQ-5D (EQ-5D-5L) utility values from FACT-G, FAACT, and FACIT-F scores to inform future cost-effectiveness analyses in the cancer cachexia setting or in lung cancer.

\section{Methods}

In performing this study, we referred to the recently published MAPS reporting statement [13] and completed the proposed 23-item checklist for mapping studies (Table A1, online appendix).

\section{Study sample}

Data from two multicenter, randomized, double blind, placebo-controlled phase 3 trials (ROMANA 1 and ROMANA 2 ) in patients with non-small cell lung cancer-cachexia (NSCLC-C) were used for this analysis. The trials were conducted at 93 sites in 19 countries between 2011 and 2014. The two studies enrolled a total of 979 patients aged $\geq 18$ years with a diagnosis of stage III or IV NSCLC and cachexia [defined as involuntary loss of $\geq 5 \%$ body weight within 6 months or body mass index (BMI) $<20 \mathrm{~kg} / \mathrm{m}^{2}$ ]; patients had an estimated life expectancy of more than 4 months at enrollment and a Eastern Cooperative Oncology Group (ECOG) performance status $\leq 2$. Details of the inclusion/exclusion criteria and the study design are described elsewhere [14]. Patients were randomly assigned 2:1 to receive active treatment (100 mg, Anamorelin HC1, Helsinn Therapeutics, Inc.) or placebo once daily over a 12-week period. Primary efficacy endpoints were the median change in lean body mass and handgrip strength over the same period. Among the secondary efficacy parameters, HRQoL was assessed by FACIT-F and FAACT (version 4) at baseline and weeks 3, 6, 9, and 12. Moreover, the EQ-5D-5L questionnaire was administered at the same time points to a subset of patients in only two sites (i.e. Poland and Hungary). For the purpose of this analysis, we used the sample of observations reporting both HRQoL instruments (i.e. FACIT-F/FAACT and EQ-5D-5L) without any distinction between the treatment arms.

\section{Instruments}

The EQ-5D-5L questionnaire is a generic, preference-based HRQoL measure comprising five domains: mobility, selfcare, usual activities, pain/discomfort, and anxiety/depression [7]. Each dimension has five levels: no problems, slight problems, moderate problems, severe problems, and extreme problems. Patients' responses to the questionnaire were 
scored using the English EQ-5D-5L value set [15], which ranges from -0.281 (state 55555) and 1 (state 11111, representing perfect health) and the Dutch EQ-5D-5L value set [16] ranging between -0.446 and 1 to illustrate the sensitivity of results to the use of alternative value sets.

The FAACT is the anorexia-cachexia-specific HRQoL instrument of the FACIT system [5]. It comprises the 27-item FACT-G and a 12-item Anorexia-Cachexia Subscale (ACS). Each item is rated on a 5-point Likert scale (from 0 to 4) ranging from "not at all" to "very much". The FAACT is the sum of the FACT-G score (0-108) and the ACS score $(0-48)$ with higher values representing better health. Similar to FAACT, the FACIT-F comprises the FACT-G and a 13-item Fatigue Subscale (0-52), yielding a total score between 0 and 160 . The FACT-G is composed of four subscales assessing physical wellbeing (PWB, 0-28), functional wellbeing (FWB, 0-28), social/family well-being (SWB, 0-28), and emotional wellbeing (EWB, 0-24). From these scales, it is also possible to calculate a Trial Outcome Index (TOI), which is the sum of PWB, FWB, and tool-specific subscales (in this study, ACS and Fatigue).

\section{Statistical analysis}

Generalized estimating equations (GEEs) were performed in order to derive mapping functions for FAACT, FACIT-F, and FACT-G. GEE is a technique facilitating the analysis of data collected in longitudinal, clustered, or repeated measures designs, which is increasingly applied in clinical trials and biomedical studies. GEE is a population-level approach based on a quasi-likelihood function that provides the population-averaged estimates of the parameters. GEEs use the generalized linear model to estimate regression parameters allowing the specification of a working correlation matrix that accounts for the type of within-subject correlation of responses on the dependent variable $[17,18]$. The GEE method was selected because of the longitudinal nature of the ROMANA trials, where repeated observations from each instrument are expected to be correlated between visits. This method has been used previously to predict EQ-5D utilities from the Functional Assessment of Cancer Therapy-Prostate (FACT-P) using data from a multicenter, randomized, placebo-controlled trial [19]. Quasi-likelihood under the independence model criterion (QIC) statistics were calculated in order to select the best-working correlation structure [20].

In order to identify the best model specification, five alternative sets of explanatory variables were compared. Models were developed in order of increasing complexity given by the level of disaggregation of FACIT questionnaires scores. In detail, EQ-5D-5L scores were predicted from the overall FACT-G, FAACT, and FACIT-F scores (Model 1), from the generic (FACT-G) and specific (ACS and Fatigue) components separately (Model 2), from the four domains that compose FACT-G (PWB, FWB, SWB, and EWB) (Model 3), from the TOI score alone (Model 4 ) and from the three components (PWB, FWB, and ACS or Fatigue) of TOI scores (Model 5). Selected clinical and demographic variables were tested for potential inclusion in the final models on the basis of their statistical significance. These variables were: age ( $>65$ or $\leq 65)$, gender, body mass index (BMI; $\leq 18.5$ or $\left.>18.5 \mathrm{~kg} / \mathrm{m}^{2}\right)$, ECOG $(0-1$ or 2$)$ performance at baseline, weight loss in previous 6 months ( $>$ or $\leq 10 \%$ of body weight) and current chemotherapy/radiotherapy status (yes or no). The great majority $(99.0 \%)$ of the patients enrolled were white, thus ethnicity was not considered as a covariate in the regression models [21]. Squared FACIT scores were tested as well to allow for nonlinear relationships with EQ-5D-5L utility. No imputation of missing values was performed in order to avoid assumptions about regarding early patient dropout. No interactions were tested, as previous research demonstrated that adding interaction terms seldom improved the model fit [11, 21, 22] and the "principle of parsimony" should be embraced in developing mapping algorithms so that they can be more readily used by future researchers [23]. Pearson's correlation was performed to estimate the degree of conceptual overlap between the source(s) and target measures that justified a mapping exercise among them (Table A2, online appendix).

\section{Model selection and cross-validation}

The performance of each model was assessed in terms of how well the responses to FACT-G, FAACT, and FACIT-F predicted EQ-5D-5L utilities. An out-of-sample validation is usually recommended to test the algorithms; however, no external datasets were available and an internal cross-validation technique was applied to derive goodness-of-fit statistics. The study sample was non-randomly divided into two groups using one-fifth (validation sample) four-fifths (development sample) split according to patients' geographical residence (i.e. Poland or Hungary). Following the approach of a previous study [24] and recommendations from the MAPS statement [13], we assumed that a non-random split ensures a more efficient validation, as the two groups are likely to differ according to some baseline characteristics. Statistical tests (i.e. Chi-squared for categorical variables and $t$ test for continuous variables) were performed in order to explore the differences between the two sub-samples. Mapping functions were fitted on the development sample, while the remaining observations were used to test the models' performance.

Model validation was performed by pooling all the visits together in order to obtain average performance indexes within the database. The mean absolute error (MAE) and root-mean square error (RMSE) were calculated to examine the differences between mean observed and predicted 
EQ-5D-5L scores, with lower values indicating better algorithm performance. The MAE is the average of absolute differences between observed and predicted utilities, while the RMSE is the root of the average of the squared differences. A paired $t$ test $(p<0.05)$ was also applied to the differences between observed and mapped EQ-5D-5L scores with significant results indicating low predictive accuracy [25]. The best performing models were selected on the basis of the lowest MAE/RMSE results. Moreover, these differences were compared to the minimal important difference (MID) in EQ-5D utility that, in cancer patients, has been estimated as 0.08 using the UK value set for the 3-level version (EQ5D-3L) [26].

All analyses were conducted using STATA version 14.1 (College Station, TX, USA) and Microsoft Excel 2013.

\section{Results}

\section{Descriptive statistics}

Demographic and baseline clinical data stratified by overall, development, and validation dataset are reported in Table 1. Overall, 96 patients completed both EQ-5D-5L and FACIT questionnaires; the number of observations per patient ranged between 1 and 5, for a total of 420 of which 332 were used to develop the algorithms and the remaining 88 for validating them. In the overall sample $(n=96)$, the majority of patients were male $(68.7 \%)$ and their mean age was 61 years; most $(86.5 \%)$ were on chemotherapy or radiotherapy treatments. The average EQ-5D-5L utility at baseline was $0.766(\mathrm{SD}=0.19)$, ranging between -0.102 and 1 (perfect health); mean FACT-G, FAAC,T and FACIT-F overall scores were $64.9(\mathrm{SD}=14.2), 93.2(\mathrm{SD}=21.0)$, and $93.2(\mathrm{SD}=22.4)$, respectively. Among FACT-G subscales, SWB had the highest score $(20.5 \pm 4.7)$, whilst EWB had the lowest $(13.4 \pm 5.0)$.

Patients in the development ( $n=76$, Poland) and validation ( $n=20$, Hungary) samples differed by BMI $\left(\mathrm{kg} / \mathrm{m}^{2}\right)$, weight loss, ECOG performance score and chemotherapy/ radiotherapy status at baseline. Significant differences $(p<0.05)$ in baseline HRQoL scores were only observed in mean EQ-5D-5L (both for English and Dutch values), FWB and Fatigue scores. Full descriptive statistics of EQ-5D-5L, FAACT and FACIT-F scores by visit for the overall sample are shown in Table 2. The distribution of EQ-5D-5L scores is shown in Fig. 1.

\section{Regression models}

Table 3a, b shows parameter estimates for all mapping functions obtained from the development sample (76 patients, 332 observations) using the English and Dutch value sets, respectively. Models with squared terms showed poorer goodness-of-fit (i.e. higher QIC) compared to models without and accordingly were not retained in the analyses (results not shown). Based on QIC results, autoregressive correlation was chosen within the GEE model by assuming that repeated measures were more strongly correlated when close together in time. The preliminary analyses testing all demographic and clinical variables are not reported; among them, only ECOG performance status (score $=2$ vs. score $=0-1$ ) was included in the final models after backward selection, with higher ECOG scores predicting lower EQ-5D-5L utility values (negative coefficient; $p<0.001$ ).

For all three FACIT instruments (FACT-G, FAACT, and FACIT-F), overall scores were highly significant $(p<0.001)$, as were the TOI scores, in models using aggregate results (Model 1 and Model 4). In models using the separate individual subscales (Model 3 and Model 5), the EWB subscale was never significantly associated with the EQ-5D-5L score; conversely, PWB had the highest explanatory value with the exception of FACIT-F Model 3, where it was not significant $(p>0.05)$. When ACS and Fatigue subscales were combined with the generic FACT-G score in models 2, the generic score was not significant in the FACIT-F model, implying that Fatigue and ECOG were sufficient to predict EQ-5D results. In all models, the coefficients of the HRQoL scales had the expected (positive) signs, indicating that better health reported by disease-specific FACT-G/FAACT/ FACIT-F tools was associated with higher EQ-5D utility. The only exception was SWB that presented a negative coefficient in all models, but its value was at the limits of statistical significance.

\section{Model selection}

A synthesis of model performances across all visits in the validation sample is reported in Table $4 \mathrm{a}$, b comparing observed and predicted EQ-5D-5L utilities using English and Dutch preference weights, respectively. Overall, the mapping algorithms predicted well. The absolute differences between mean observed and mean predicted EQ5D-5L utilities were far below the MID of 0.08 reported for EQ-5D-3L in cancer studies [26]. Moreover, none of the estimates fell outside the theoretical range of EQ-5D-5L (i.e. $-0.281,1.000$ ) with the exception of FACIT-F Model 3 (UK), but only just (i.e. 1.014). Most of the t-test comparisons between observed and mapped scores yielded a non-significant $p$ value $(<0.05)$. However, all differences between observed and predicted values were negative, due to an overall tendency towards over-prediction in the poorest health states (EQ-5D-5L utility $\leq 0.700)$. At the same time, the range of predicted EQ-5D-5L utilities was generally narrower than the observed values and the algorithms failed to predict the value of 1 corresponding to perfect 
Table 1 Sample characteristics and HRQoL scores (at baseline) stratified by study dataset

\begin{tabular}{|c|c|c|c|c|c|}
\hline Variable & Statistics & Total sample $(n=96)$ & $\begin{array}{l}\text { Development } \\
\text { sample }(n=76)\end{array}$ & $\begin{array}{l}\text { Validation sam- } \\
\text { ple }(n=20)\end{array}$ & $p$ value \\
\hline \multicolumn{6}{|l|}{ Demographic and clinical data } \\
\hline Country & $\begin{array}{l}\text { Poland }[N(\%)] \\
\text { Hungary }[N(\%)]\end{array}$ & $\begin{array}{l}76(79.2) \\
20(20.8)\end{array}$ & $\begin{array}{l}76(100.0) \\
0(0.0)\end{array}$ & $\begin{array}{l}0(0.0) \\
20(100.0)\end{array}$ & \\
\hline Age (years) & $\begin{array}{l}\text { Mean }( \pm \mathrm{SD}) \\
\text { Range (min, max })\end{array}$ & $\begin{array}{l}61.1( \pm 8.7) \\
36,85\end{array}$ & $\begin{array}{l}61.3( \pm 9.0) \\
36,85\end{array}$ & $\begin{array}{l}60.5( \pm 7.7) \\
46,80\end{array}$ & $0.7217^{\mathrm{a}}$ \\
\hline Gender & Male $[N(\%)]$ & $66(68.7)$ & $54(71.0)$ & $12(60.0)$ & $0.343^{\mathrm{b}}$ \\
\hline BMI $\left(\mathrm{Kg} / \mathrm{m}^{2}\right)$ & $\leq 18.5[N(\%)]$ & $7(7.29)$ & $3(3.95)$ & $4(20.0)$ & $0.014^{\mathrm{b}^{*}}$ \\
\hline Weight loss (in previous 6 months) & $\begin{array}{l}>10 \% \text { of body weight } \\
{[N(\%)]}\end{array}$ & 39 (40.6) & $25(32.9)$ & $14(70.0)$ & $0.003^{\mathrm{b}, * *}$ \\
\hline ECOG & $\begin{array}{l}0-1[N(\%)] \\
2[N(\%)]\end{array}$ & $\begin{array}{l}78(81.2) \\
18(18.8)\end{array}$ & $\begin{array}{l}58(76.3) \\
18(23.7)\end{array}$ & $\begin{array}{l}20(100.0) \\
0(0.0)\end{array}$ & $0.016^{\mathrm{b}, *}$ \\
\hline On chemotherapy/radiotherapy & Yes $[N(\%)]$ & $83(86.5)$ & $74(97.4)$ & $9(45.0)$ & $0.000^{\mathrm{b}, * * *}$ \\
\hline \multicolumn{6}{|l|}{ HRQoL scores (at baseline) } \\
\hline FAACT & $\begin{array}{l}\text { Mean }( \pm \text { SD }) \\
\text { Range (min, max })\end{array}$ & $\begin{array}{l}93.2( \pm 21.0) \\
28.0,141.0\end{array}$ & $\begin{array}{l}93.4( \pm 21.8) \\
28.0,141.0\end{array}$ & $\begin{array}{l}92.6( \pm 17.8) \\
47.8,129.0\end{array}$ & $0.8774^{\mathrm{a}}$ \\
\hline FACIT-F & $\begin{array}{l}\text { Mean }( \pm \mathrm{SD}) \\
\text { Range (min, max })\end{array}$ & $\begin{array}{l}93.2( \pm 22.4) \\
20.0,140.0\end{array}$ & $\begin{array}{l}92.6( \pm 23.2) \\
20.0,140.0\end{array}$ & $\begin{array}{l}95.4( \pm 19.6) \\
57.8,134.0\end{array}$ & $0.6274^{\mathrm{a}}$ \\
\hline FACT-G & $\begin{array}{l}\text { Mean }( \pm \mathrm{SD}) \\
\text { Range (min, max })\end{array}$ & $\begin{array}{l}64.9( \pm 14.2) \\
18.0,95.0\end{array}$ & $\begin{array}{l}65.4( \pm 14.7) \\
18.0,95.0\end{array}$ & $\begin{array}{l}63.0( \pm 12.0) \\
36.8,88.0\end{array}$ & $0.5086^{\mathrm{a}}$ \\
\hline PWB & $\begin{array}{l}\text { Mean }( \pm \mathrm{SD}) \\
\text { Range (min, max) }\end{array}$ & $\begin{array}{l}16.2( \pm 5.1) \\
4.0,28.0\end{array}$ & $\begin{array}{l}15.7( \pm 5.2) \\
4.0,28.0\end{array}$ & $\begin{array}{l}17.8( \pm 4.3) \\
11.0,27.0\end{array}$ & $0.0976^{\mathrm{a}}$ \\
\hline FWB & $\begin{array}{l}\text { Mean }( \pm \mathrm{SD}) \\
\text { Range (min, max })\end{array}$ & $\begin{array}{l}14.8( \pm 5.3) \\
1.0,26.0\end{array}$ & $\begin{array}{l}15.8( \pm 4.9) \\
3.0,26.0\end{array}$ & $\begin{array}{l}11.0( \pm 5.4) \\
1.0,21.0\end{array}$ & $0.0003^{\mathrm{a}, * *}$ \\
\hline SWB & $\begin{array}{l}\text { Mean }( \pm \mathrm{SD}) \\
\text { Range (min, max })\end{array}$ & $\begin{array}{l}20.5( \pm 4.7) \\
4.0,28.0\end{array}$ & $\begin{array}{l}20.6( \pm 5.0) \\
4.0,28.0\end{array}$ & $\begin{array}{l}19.8( \pm 3.5) \\
12.8,26.0\end{array}$ & $0.4864^{\mathrm{a}}$ \\
\hline EWB & $\begin{array}{l}\text { Mean }( \pm \mathrm{SD}) \\
\text { Range (min, max) }\end{array}$ & $\begin{array}{l}13.4( \pm 5.0) \\
0.0,24.0\end{array}$ & $\begin{array}{l}13.2( \pm 5.4) \\
0.0,24.0\end{array}$ & $\begin{array}{l}14.3( \pm 3.5) \\
7.0,21.0\end{array}$ & $0.3800^{\mathrm{a}}$ \\
\hline FAACT (ACS) & $\begin{array}{l}\text { Mean }( \pm \mathrm{SD}) \\
\text { Range (min, max) }\end{array}$ & $\begin{array}{l}28.3( \pm 9.0) \\
5.0,46.0\end{array}$ & $\begin{array}{l}28.0( \pm 9.4) \\
5.0,46.0\end{array}$ & $\begin{array}{l}29.5( \pm 7.4) \\
11.0,43.0\end{array}$ & $0.4957^{\mathrm{a}}$ \\
\hline FACIT-F (fatigue) & $\begin{array}{l}\text { Mean }( \pm \mathrm{SD}) \\
\text { Range (min, max) }\end{array}$ & $\begin{array}{l}28.3( \pm 9.8) \\
2.0,49.0\end{array}$ & $\begin{array}{l}27.2( \pm 9.9) \\
2.0,49.0\end{array}$ & $\begin{array}{l}32.3( \pm 8.2) \\
17.0,46.0\end{array}$ & $0.0360^{\mathrm{a}, *}$ \\
\hline FAACT (TOI) & $\begin{array}{l}\text { Mean }( \pm \mathrm{SD}) \\
\text { Range (min, max })\end{array}$ & $\begin{array}{l}59.3( \pm 15.9) \\
23.0,99.0\end{array}$ & $\begin{array}{l}59.5( \pm 16.3) \\
23.0,99.0\end{array}$ & $\begin{array}{l}58.4( \pm 14.5) \\
26.0,85.0\end{array}$ & $0.7840^{\mathrm{a}}$ \\
\hline FACIT-F (TOI) & $\begin{array}{l}\text { Mean }( \pm \mathrm{SD}) \\
\text { Range (min, max) }\end{array}$ & $\begin{array}{l}59.3( \pm 16.9) \\
15.0,98.0\end{array}$ & $\begin{array}{l}58.8( \pm 17.2) \\
15.0,98.0\end{array}$ & $\begin{array}{l}61.2( \pm 16.1) \\
36.0,90.0\end{array}$ & $0.5643^{\mathrm{a}}$ \\
\hline EQ-5D-5L (UK index) & $\begin{array}{l}\text { Mean }( \pm \mathrm{SD}) \\
\text { Range (min, max) }\end{array}$ & $\begin{array}{l}0.766( \pm 0.19) \\
-0.102,1.000\end{array}$ & $\begin{array}{l}0.746( \pm 0.20) \\
-0.102,1.000\end{array}$ & $\begin{array}{l}0.841( \pm 0.13) \\
0.527,1.000\end{array}$ & $0.0478^{\mathrm{a}, *}$ \\
\hline EQ-5D-5L (NL index) & $\begin{array}{l}\text { Mean }( \pm \mathrm{SD}) \\
\text { Range (min, max) }\end{array}$ & $\begin{array}{l}0.702( \pm 0.23) \\
-0.289,1.000\end{array}$ & $\begin{array}{l}0.678( \pm 0.25) \\
-0.289,1.000\end{array}$ & $\begin{array}{l}0.792( \pm 0.15) \\
0.383,1.000\end{array}$ & $0.0514^{\mathrm{a}, *}$ \\
\hline
\end{tabular}

$S D$ standard deviation; BMI body mass index, ECOG Eastern Cooperative Oncology Group, FACT-G functional assessment of cancer therapygeneral, FAACT functional assessment of anorexia/cachexia treatment, FACIT-F functional assessment of chronic illness therapy-fatigue, $P W B$ physical wellbeing, $F W B$ functional wellbeing, EWB emotional wellbeing, SWB social/family wellbeing, ACS Anorexia-Cachexia Subscale, TOI trial outcome index, $E Q-5 D-5 L$ EuroQol five-dimension five-level, $U K$ United Kingdom, $N L$ Netherlands

${ }^{*} p$ value $<0.05$

$* * p$ value $<0.01$

$* * * p$ value $<0.001$

${ }^{\mathrm{a}} T$ test

${ }^{\mathrm{b}} \mathrm{Chi}$-square test

health especially when using FACT-G and FAACT, whilst considerably larger intervals were obtained through FACITF regression models.
Best performing algorithms were identified for each FACIT questionnaire according to the lowest MAE/RMSE scores, namely Models 3 for FACT-G and FAACT, and 
Table 2 Descriptive statistics of HRQoL observed scores by study visits (overall sample)

\begin{tabular}{|c|c|c|c|c|c|c|}
\hline Instrument & Statistics & Baseline & Week 3 & Week 6 & Week 9 & Week 12 \\
\hline \multirow{4}{*}{$\begin{array}{l}\text { FAACT (overall) } \\
\text { Theoretical range (min, max): 0, } 156\end{array}$} & $N$ & 96 & 89 & 85 & 78 & 76 \\
\hline & Mean $( \pm S D)$ & $93.2( \pm 21.0)$ & $100.5( \pm 22.6)$ & $101.5( \pm 21.2)$ & $100.3( \pm 20.7)$ & $95.3( \pm 23.6)$ \\
\hline & Range (min, $\max$ ) & $28.0,141.0$ & $57.0,148.0$ & $41.0,147.7$ & $55.3,152.0$ & $45.5-156.0$ \\
\hline & $\%$ floor, $\%$ ceiling & $0.0,0.0$ & $0.0,0.0$ & $0.0,0.0$ & $0.0,0.0$ & $0.0,1.3$ \\
\hline \multirow{4}{*}{$\begin{array}{l}\text { FACIT-F (overall) } \\
\text { Theoretical range (min, max): } 0,160\end{array}$} & $N$ & 96 & 89 & 85 & 78 & 75 \\
\hline & Mean $( \pm$ SD $)$ & $93.2( \pm 22.4)$ & $99.2( \pm 24.4)$ & $99.1( \pm 23.0)$ & $97.1( \pm 21.9)$ & $92.0( \pm 26.2)$ \\
\hline & Range (min, max) & $20.0,140.0$ & $34.3,154.0$ & $37.0,146.0$ & $51.3,156.0$ & $13.5,160.0$ \\
\hline & $\%$ floor, \% ceiling & $0.0,0.0$ & $0.0,0.0$ & $0.0,0.0$ & $0.0,0.0$ & $0.0,1.3$ \\
\hline \multirow{4}{*}{$\begin{array}{l}\text { FACT-G } \\
\text { Theoretical range (min, max): 0, } 108\end{array}$} & $N$ & 96 & 89 & 85 & 78 & 76 \\
\hline & Mean $( \pm S D)$ & $64.9( \pm 14.2)$ & $69.1( \pm 15.4)$ & $69.3( \pm 14.1)$ & $68.2( \pm 13.7)$ & $64.6( \pm 16.3)$ \\
\hline & Range (min, $\max$ ) & $18.0,95.0$ & $31.3,102.0$ & $33.0,101.7$ & $41.0,104.0$ & $12.5,108.0$ \\
\hline & $\%$ floor, $\%$ ceiling & $0.0,0.0$ & $0.0,0.0$ & $0.0,0.0$ & $0.0,0.0$ & $0.0,1.3$ \\
\hline \multirow{4}{*}{$\begin{array}{l}\text { PWB } \\
\text { Theoretical range (min, max): } 0,28\end{array}$} & $N$ & 96 & 90 & 86 & 78 & 76 \\
\hline & Mean $( \pm$ SD $)$ & $16.2( \pm 5.1)$ & $17.1( \pm 5.6)$ & $17.5( \pm 5.8)$ & $17.1( \pm 5.3)$ & $16.3( \pm 5.9)$ \\
\hline & Range (min, $\max$ ) & $4.0,28.0$ & $1.0,28.0$ & $1.0,28.0$ & $3.0,28.0$ & $0.0,28.0$ \\
\hline & $\%$ floor, \% ceiling & $0.0,1.0$ & $0.0,2.2$ & $0.0,2.3$ & $0.0,3.8$ & $1.3,3.9$ \\
\hline \multirow{4}{*}{$\begin{array}{l}\text { FWB } \\
\text { Theoretical range (min, } \max ): 0,28\end{array}$} & $N$ & 96 & 90 & 85 & 78 & 76 \\
\hline & Mean $( \pm S D)$ & $14.8( \pm 5.3)$ & $15.8( \pm 5.5)$ & $15.7( \pm 5.4)$ & $15.5( \pm 4.7)$ & $14.7( \pm 5.4)$ \\
\hline & Range (min, max) & $1.0,26.0$ & $0.0,27.0$ & $3.0,28.0$ & $4.0,27.0$ & $2.0,28.0$ \\
\hline & $\%$ floor, $\%$ ceiling & $0.0,0.0$ & $1.1,0.0$ & $0.0,1.2$ & $0.0,0.0$ & $0.0,1.3$ \\
\hline \multirow{4}{*}{$\begin{array}{l}\text { SWB } \\
\text { Theoretical range (min, max): } 0,28\end{array}$} & $N$ & 96 & 90 & 86 & 78 & 76 \\
\hline & Mean $( \pm S D)$ & $20.5( \pm 4.7)$ & $21.1( \pm 4.4)$ & $20.9( \pm 4.2)$ & $20.6( \pm 4.4)$ & $20.4( \pm 4.2)$ \\
\hline & Range (min, max) & $4.0,28.0$ & $9.3,28.0$ & $8.0,28.0$ & $9.0,28.0$ & $10.5,28.0$ \\
\hline & $\%$ floor, $\%$ ceiling & $0.0,5.2$ & $0.0,8.9$ & $0.0,5.8$ & $0.0,5.1$ & $0.0,10.5$ \\
\hline \multirow{4}{*}{$\begin{array}{l}\text { EWB } \\
\text { Theoretical range (min, } \max ): 0,24\end{array}$} & $N$ & 96 & 89 & 85 & 78 & 76 \\
\hline & Mean $( \pm S D)$ & $13.4( \pm 5.0)$ & $14.9( \pm 4.7)$ & $15.1( \pm 4.5)$ & $14.9( \pm 4.3)$ & $13.3( \pm 5.4)$ \\
\hline & Range (min, max) & 0,24 & 4,24 & 1,23 & 6,24 & 0,24 \\
\hline & $\%$ floor, \% ceiling & $2.1,2.1$ & $0.0,1.1$ & $0.0,0.0$ & $0.0,1.3$ & $1.3,2.6$ \\
\hline \multirow{4}{*}{$\begin{array}{l}\text { FAACT (ACS) } \\
\text { Theoretical range (min, } \max ): 0,48\end{array}$} & $N$ & 96 & 90 & 86 & 78 & 76 \\
\hline & Mean $( \pm S D)$ & $28.3( \pm 9.0)$ & $31.4( \pm 9.3)$ & $32.1( \pm 8.4)$ & $32.2( \pm 8.9)$ & $30.7( \pm 9.3)$ \\
\hline & Range (min, max) & $5.0,46.0$ & $8.0,47.0$ & $8.0,47.0$ & $10.0,48.0$ & $8.0,48.0$ \\
\hline & $\%$ floor, \% ceiling & $0.0,0.0$ & $0.0,0.0$ & $0.0,0.0$ & $0.0,1.3$ & $0.0,2.6$ \\
\hline \multirow{4}{*}{$\begin{array}{l}\text { FACIT-F (Fatigue) } \\
\text { Theoretical range (min, max): } 0,52\end{array}$} & $N$ & 96 & 90 & 86 & 78 & 75 \\
\hline & Mean $( \pm S D)$ & $28.3( \pm 9.8)$ & $30.2( \pm 10.8)$ & $29.8( \pm 10.4)$ & $29.0( \pm 10.1)$ & $27.8( \pm 10.9)$ \\
\hline & Range (min, max) & $2.0,49.0$ & $3.0,52.0$ & $1.0,50.0$ & $6.0,52.0$ & $1.0,52.0$ \\
\hline & $\%$ floor, $\%$ ceiling & $0.0,0.0$ & $0.0,1.1$ & $0.0,0.0$ & $0.0,2.6$ & $0.0,1.3$ \\
\hline \multirow{4}{*}{$\begin{array}{l}\text { FAACT (TOI) } \\
\text { Theoretical range (min, max): 0, } 104\end{array}$} & $N$ & 96 & 89 & 85 & 78 & 76 \\
\hline & Mean $( \pm \mathrm{SD})$ & $59.32( \pm 15.88)$ & $64.51( \pm 17.16)$ & $65.44( \pm 16.71)$ & $64.79( \pm 15.68)$ & $61.60( \pm 17.46)$ \\
\hline & Range (min, max) & 23,99 & 24,98 & 12,100 & 21,102 & 28,104 \\
\hline & $\%$ floor, \% ceiling & $0.0,0.0$ & $0.0,0.0$ & $0.0,0.0$ & $0.0,0.0$ & $0.0,1.3$ \\
\hline \multirow{4}{*}{$\begin{array}{l}\text { FACIT-F (TOI) } \\
\text { Theoretical range (min, max): 0, } 108\end{array}$} & $N$ & 96 & 89 & 85 & 78 & 75 \\
\hline & Mean $( \pm \mathrm{SD})$ & $59.29( \pm 16.95)$ & $63.26( \pm 18.98)$ & $63.06( \pm 18.47)$ & $61.61( \pm 17.13)$ & $58.47( \pm 19.89$ \\
\hline & Range (min, max) & 15,98 & 13,104 & 8,98 & 17,106 & 3,108 \\
\hline & $\%$ floor, $\%$ ceiling & $0.0,0.0$ & $0.0,0.0$ & $0.0,0.0$ & $0.0,0.0$ & $0.0,1.3$ \\
\hline \multirow{4}{*}{$\begin{array}{l}\text { EQ-5D-5L utility (UK index) } \\
\text { Theoretical range (min, max): }-0.281 \text {, } \\
\quad 1\end{array}$} & $N$ & 96 & 88 & 85 & 77 & 74 \\
\hline & Mean $( \pm S D)$ & $0.766( \pm 0.19)$ & $0.792( \pm 0.18)$ & $0.790( \pm 0.19)$ & $0.794( \pm 0.16)$ & $0.760( \pm 0.22)$ \\
\hline & Range (min, max) & $-0.102,1.000$ & $0.103,1.000$ & $-0.218,1.000$ & $0.338,1.000$ & $-0.213,1.000$ \\
\hline & $\%$ floor, $\%$ ceiling & $0.0,10.4$ & $0.0,18.2$ & $0.0,18.8$ & $0.0,18.2$ & $0.0,17.6$ \\
\hline
\end{tabular}


Table 2 (continued)

\begin{tabular}{lllllll}
\hline Instrument & Statistics & Baseline & Week 3 & Week 6 & Week 9 & Week 12 \\
\hline EQ-5D-5L utility (NL index) & $N$ & 96 & 88 & 85 & 77 & 74 \\
Theoretical range (min, max): -0.446, & Mean $( \pm$ SD) & $0.702( \pm 0.23)$ & $0.736( \pm 0.22)$ & $0.744( \pm 0.23)$ & $0.752( \pm 0.19)$ & $0.709( \pm 0.25)$ \\
1 & Range (min, max) & $-0.289,1.000$ & $-0.077,1.000$ & $-0.391,1.000$ & $0.195,1.000$ & $-0.326,1.000$ \\
& \% floor, \% ceiling & $0.0,10.4$ & $0.0,18.2$ & $0.0,18.8$ & $0.0,18.2$ & $0.0,17.6$ \\
\hline
\end{tabular}

$S D$ standard deviation, FACT-G Functional assessment of cancer therapy-general, FACIT functional assessment of chronic illness therapy, $F A A C T$ functional assessment of anorexia/cachexia treatment, $F A C I T-F$ functional assessment of chronic illness therapy-fatigue, $P W B$ physical wellbeing, $F W B$ functional wellbeing, $E W B$ emotional wellbeing, $S W B$ social/family wellbeing, ACS Anorexia-Cachexia Subscale, TOI trial outcome index, EQ-5D-5L EuroQol five-dimension five-level, $U K$ United Kingdom, $N L$ Netherlands

Model 4 for FACIT-F (both for English and Dutch values). Scatterplots displaying observed and predicted EQ-5D-5L utility scores for the three best performing models applied to the validation sample (20 patients, 88 observations) are shown in Fig. 1a, b.

\section{Discussion}

In the absence of EQ-5D or other preference-based HRQoL measurements, mapping is a useful tool in order to estimate utility values to be adopted in cost-effectiveness analyses. Over the past decade, there has been a rapid increase of mapping studies predicting generic preference-based scores from non-preference based, disease-specific results. In the area of oncology, eight studies have mapped EQ-5D from the FACIT questionnaires: three studies [11, 22,27] mapped the general FACT-G version in various types of cancer (including lung cancer); another three algorithms [19, 21, 28] used the prostate-specific (FACT-P) module, while the remaining two adopted the melanoma-specific (FACT-M) [29] and breast-specific (FACT-B) [30] versions. Until now, no algorithms were available to map EQ-5D scores from FAACT, which is the FACIT tool specifically aimed at measuring HRQoL in patients with cancer cachexia; nor is there a mapping algorithm for the FACIT-F. Moreover, the existing algorithms map to the EQ-5D-3L rather than the EQ-5D-5L; thus, it was not possible to apply these functions to our database of EQ-5D-5L utilities. In addition, most mapping studies have used cross-sectional data and Ordinary Least Square (OLS) regression in modeling FACIT scores; even when data from multiple time points were available, only baseline information were used [21] or repeated observations were pooled together in order to increase the sample size [31].

This study, following the approach of a previous one [19], estimated GEE models to account for the longitudinal nature of the data. To increase the usability of the mapping algorithms, common demographics (e.g. age) and clinical variables (e.g. BMI), which are likely to be collected in clinical studies dealing with cancer cachexia or NSCLC were initially tested in the models. Among them, only ECOG performance score displayed a significant coefficient $(p<0.05)$ and was retained in the final models. Moreover, in addition to developing a mapping function to predict EQ-5D-5L utilities from FAACT and FACIT-F, we provided separate algorithms for the general instrument (FACT-G). Disaggregated models predicting EQ-5D-5L utility values from individual FACIT subscales were found to have better predictive ability in the case of FACT-G and FAACT, while the best performing algorithm for FACIT-F was that modeling the TOI score. These results are aligned with the current mapping literature which has shown greater explanatory power from regression models using disaggregated information instead of summary scores from a disease-specific measure [22]. Nevertheless, all the algorithms performed quite well; overall, MAE and RMSE values were comparable across the models and lower than those reported by other mapping studies [19, $24,32]$. No considerable differences were found between the algorithms developed using the English and the Dutch value sets, which identified the same best performing models (Model 3, Model 3, Model 4) within each FACIT instrument; however, mapping using English weights performed slightly better in terms of lower MAE/RMSE and smaller differences between mean observed and mean predicted EQ5D-5L utilities. The estimated coefficients are aligned with those reported by a previous study [11] mapping FACT$\mathrm{G}$ in cancer patients affected by breast, colorectal or lung cancer, where regression coefficients for the overall score were between 0.005 and 0.008 (according to the technique adopted) and coefficients for individual subscales fell in the interval 0.006-0.013 for PWB, 0.005-0.010 for FWB, 0.002-0.008 for EWB. Our slightly lower estimates may be a consequence of the different regression method (GEEs) applied, or possibly is reflecting differences in the patient groups, for example, in terms of severity.

This study presents a few limitations. First, in calculating EQ-5D-5L utilities, we adopted country preference weights that may not be the best estimates for Hungarian and Polish populations. However, due to unavailability of EQ-5D-5L tariffs for these two countries, we selected the 
(a)

FACT-G Model 3

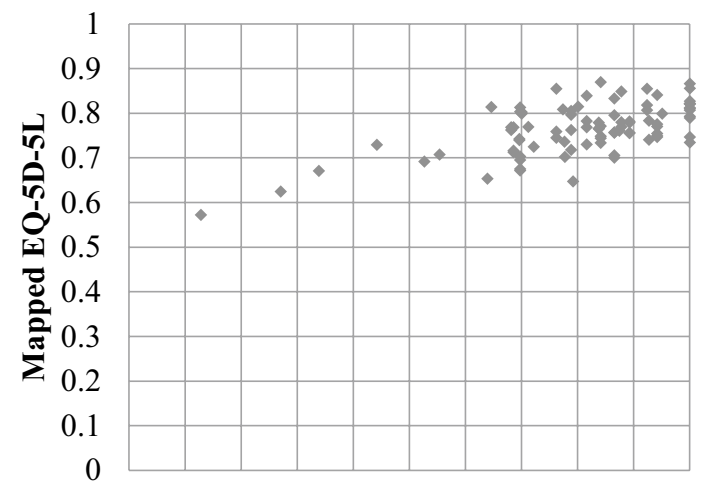

$\begin{array}{llllllllllll}0.0 & 0.1 & 0.2 & 0.3 & 0.4 & 0.5 & 0.6 & 0.7 & 0.8 & 0.9 & 1.0\end{array}$

Observed EQ-5D-5L

FAACT Model 3

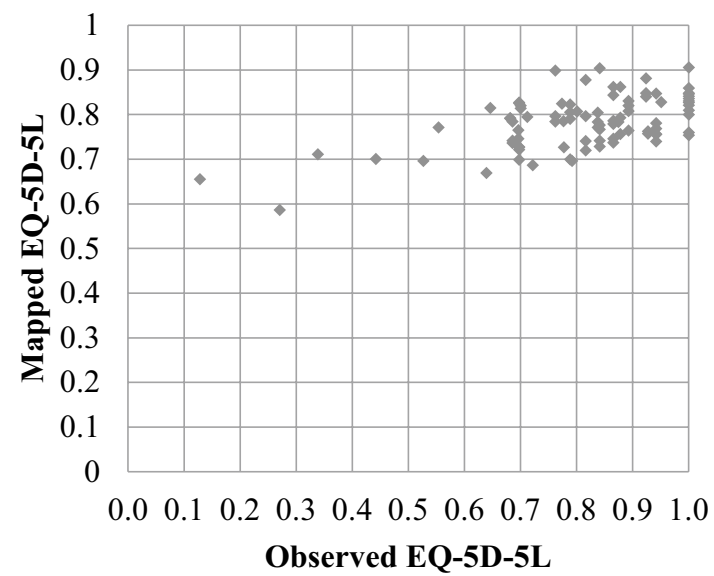

FACIT-F Model 4

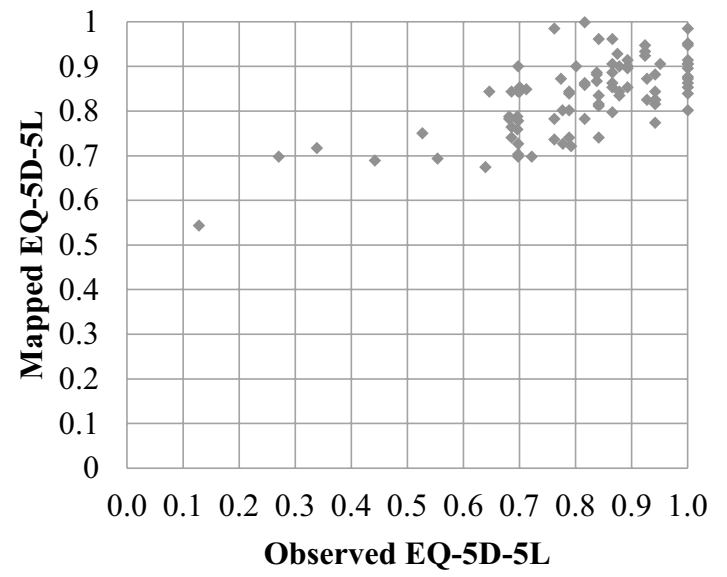

Fig. 1 a Scatterplot of observed versus predicted EQ-5D-5L utility across all visits using the best performing models (validation sample, UK weights). b Scatterplot of observed versus predicted EQ-5D-5L (b)

FACT-G Model 3

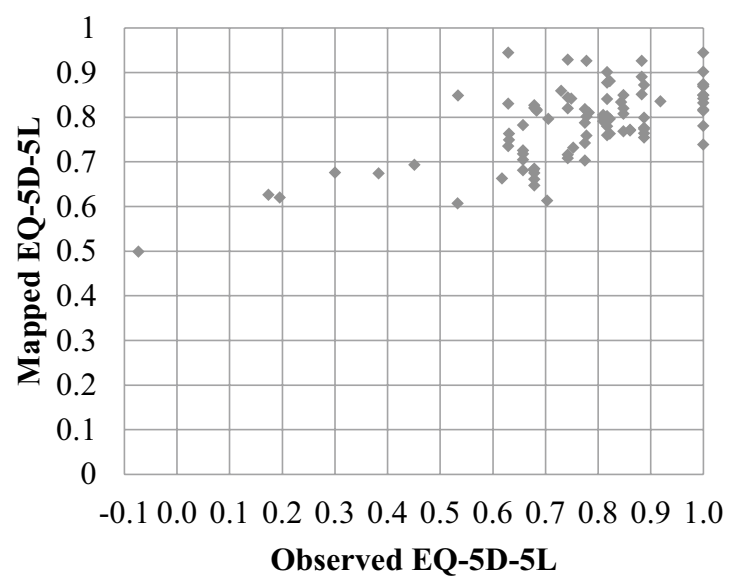

FAACT Model 3

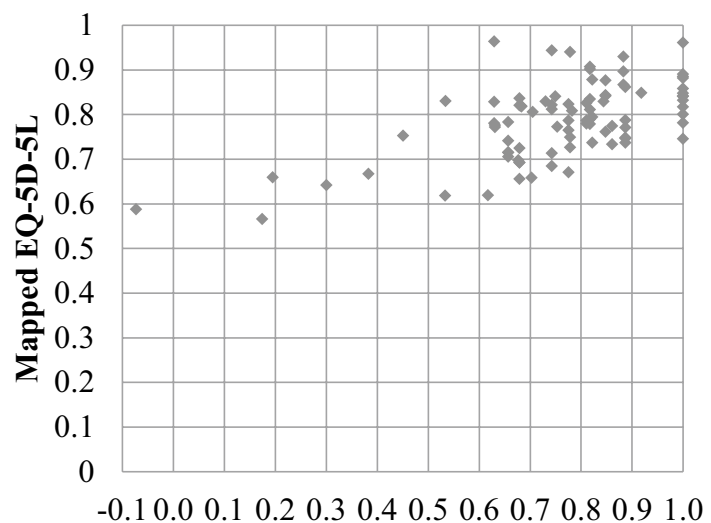

Observed EQ-5D-5L

\section{FACIT-F Model 4}

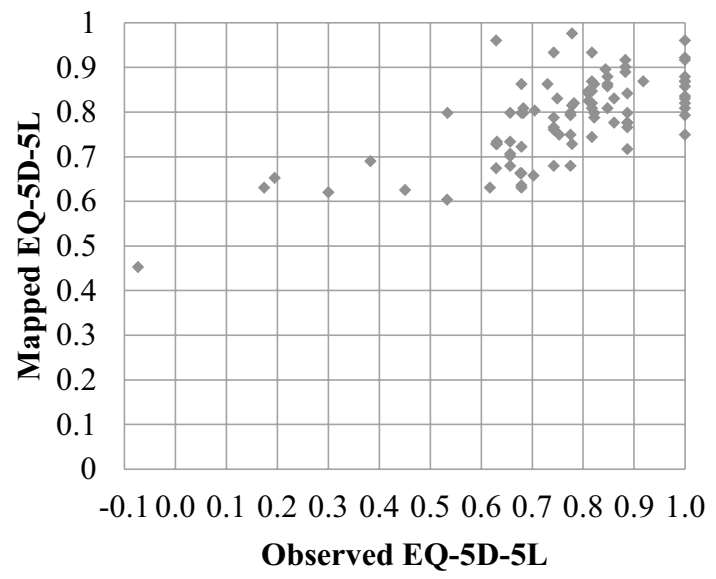

utility across all visits using the best performing models (validation sample, NL weights) 
Table 3 GEE coefficient estimates (development sample)

\begin{tabular}{|c|c|c|c|c|c|c|c|c|c|c|}
\hline & \multicolumn{2}{|l|}{ Model 1} & \multicolumn{2}{|l|}{ Model 2} & \multicolumn{2}{|l|}{ Model 3} & \multicolumn{2}{|l|}{ Model 4} & \multicolumn{2}{|l|}{ Model 5} \\
\hline & $\beta$ & SE & $\beta$ & SE & $\beta$ & SE & $\beta$ & SE & $\beta$ & SE \\
\hline \multicolumn{11}{|c|}{ (a) UK weights } \\
\hline \multicolumn{11}{|c|}{ FACT-G } \\
\hline Intercept & $0.5387 * * *$ & 0.0485 & - & - & $0.6185 * * *$ & 0.0517 & - & - & & \\
\hline FACT-G & $0.0041 * * *$ & 0.0007 & - & - & & & - & - & & \\
\hline PWB & & & - & - & $0.0094 * * *$ & 0.0019 & - & - & & \\
\hline FWB & & & - & - & $0.0052 * *$ & 0.0019 & - & - & & \\
\hline SWB & & & - & - & $-0.0044^{*}$ & 0.0022 & - & - & & \\
\hline EWB & & & - & - & 0.0035 & 0.0022 & - & - & & \\
\hline ECOG & $-0.1865 * * *$ & 0.0321 & - & - & $-0.1586^{* * *}$ & 0.0310 & - & - & & \\
\hline \multicolumn{11}{|l|}{ FAACT } \\
\hline Intercept & $0.4952 * * *$ & 0.0467 & $0.5088 * * *$ & 0.0474 & $0.5841 * * *$ & 0.0524 & $0.5210 * * *$ & 0.0392 & $0.5240 * * *$ & 0.0390 \\
\hline FAACT & $0.0033 * * *$ & 0.0004 & & & & & & & & \\
\hline FACT-G & & & $0.0022 * *$ & 0.0008 & & & & & & \\
\hline PWB & & & & & $0.0071 * *$ & 0.0021 & & & $0.0077 * * *$ & 0.0020 \\
\hline FWB & & & & & $0.0040^{*}$ & 0.0020 & & & 0.0030 & 0.0018 \\
\hline SWB & & & & & -0.0042 & 0.0022 & & & & \\
\hline EWB & & & & & 0.0021 & 0.0023 & & & & \\
\hline ACS & & & $0.0051 * * *$ & 0.0013 & $0.0035^{* *}$ & 0.0013 & & & $0.0037 * *$ & 0.0013 \\
\hline TOI & & & & & & & $0.0046^{* * *}$ & 0.0006 & & \\
\hline ECOG & $-0.1738 * * *$ & 0.0310 & $-0.1702 * * *$ & 0.0308 & $-0.1524 * * *$ & 0.0304 & $-0.1607 * * *$ & 0.0305 & $-0.1584 * * *$ & 0.0304 \\
\hline \multicolumn{11}{|l|}{ FACIT-F } \\
\hline Intercept & $0.5000 * * *$ & 0.0433 & $0.5604 * * *$ & 0.0461 & $0.6157 * * *$ & 0.0503 & $0.5290 * * *$ & 0.0357 & $0.5442 * * *$ & 0.0363 \\
\hline FACIT-F & $0.0033 * * *$ & 0.0004 & & & & & & & & \\
\hline FACT-G & & & 0.0006 & 0.0009 & & & & & & \\
\hline PWB & & & & & 0.0041 & 0.0022 & & & $0.0045^{*}$ & 0.0022 \\
\hline FWB & & & & & 0.0029 & 0.0020 & & & 0.0017 & 0.0018 \\
\hline SWB & & & & & $-0.0048^{*}$ & 0.0021 & & & & \\
\hline EWB & & & & & 0.0010 & 0.0022 & & & & \\
\hline Fatigue & & & $0.0074 * * *$ & 0.0013 & $0.0059 * * *$ & 0.0014 & & & $0.0058 * * *$ & 0.0013 \\
\hline TOI & & & & & & & $0.0047 * * *$ & 0.0005 & & \\
\hline ECOG & $-0.1711 * * *$ & 0.0310 & $-0.1611 * * *$ & 0.0307 & $-0.1484 * * *$ & 0.0305 & $-0.1568 * * *$ & 0.0305 & $-0.1553 * * *$ & 0.0303 \\
\hline \multicolumn{11}{|c|}{ (b) NL weights } \\
\hline \multicolumn{11}{|l|}{ FACT-G } \\
\hline Intercept & $0.4443 * * *$ & 0.0569 & & & $0.5390 * * *$ & 0.0609 & & & & \\
\hline FACT-G & $0.0049 * * *$ & 0.0008 & & & & & & & & \\
\hline PWB & & & & & $0.0107 * * *$ & 0.0022 & & & & \\
\hline FWB & & & & & $0.0066^{* *}$ & 0.0023 & & & & \\
\hline SWB & & & & & $-0.0051^{*}$ & 0.0026 & & & & \\
\hline EWB & & & & & 0.0037 & 0.0026 & & & & \\
\hline ECOG & $-0.2225 * * *$ & 0.0385 & & & $-0.1906 * * *$ & 0.0377 & & & & \\
\hline \multicolumn{11}{|l|}{ FAACT } \\
\hline Intercept & $0.3956 * * *$ & 0.0549 & $0.4098 * * *$ & 0.0558 & $0.4993 * * *$ & 0.0618 & $0.4263 * * *$ & 0.0462 & $0.4291 * * *$ & 0.0459 \\
\hline FAACT & $0.0038 * * *$ & 0.0005 & & & & & & & & \\
\hline FACT-G & & & $0.0027 * *$ & 0.0010 & & & & & & \\
\hline PWB & & & & & $0.0082 * *$ & 0.0024 & & & $0.0088 * * *$ & 0.0023 \\
\hline FWB & & & & & $0.0052 *$ & 0.0023 & & & 0.0040 & 0.0021 \\
\hline SWB & & & & & -0.0048 & 0.0025 & & & & \\
\hline EWB & & & & & 0.0021 & 0.0026 & & & & \\
\hline
\end{tabular}


Table 3 (continued)

\begin{tabular}{|c|c|c|c|c|c|c|c|c|c|c|}
\hline & \multicolumn{2}{|l|}{ Model 1} & \multicolumn{2}{|l|}{ Model 2} & \multicolumn{2}{|l|}{ Model 3} & \multicolumn{2}{|l|}{ Model 4} & \multicolumn{2}{|l|}{ Model 5} \\
\hline & $\beta$ & SE & $\beta$ & SE & $\beta$ & SE & $\beta$ & SE & $\beta$ & SE \\
\hline ACS & & & $0.0057 * * *$ & 0.0015 & $0.0039 *$ & 0.0015 & & & $0.0042 * *$ & 0.0015 \\
\hline TOI & & & & & & & $0.0054 * * *$ & 0.0007 & & \\
\hline ECOG & $-0.2078 * * *$ & 0.0372 & $-0.2040 * * *$ & 0.0371 & $-0.1834 * * *$ & 0.0370 & $-0.1925^{* * *}$ & 0.0368 & $-0.1901 * * *$ & 0.0367 \\
\hline \multicolumn{11}{|l|}{ FACIT-F } \\
\hline Intercept & $0.4019 * * *$ & 0.0510 & $0.4673 * * *$ & 0.0543 & $0.5350 * * *$ & 0.0593 & $0.4364 * * *$ & 0.0421 & $0.4520 * * *$ & 0.0428 \\
\hline FACIT-F & $0.0038 * * *$ & 0.0005 & & & & & & & & \\
\hline FACT-G & & & 0.0010 & 0.0010 & & & & & & \\
\hline PWB & & & & & 0.0048 & 0.0026 & & & $0.0054 *$ & 0.0025 \\
\hline FWB & & & & & 0.0040 & 0.0023 & & & 0.0026 & 0.0021 \\
\hline SWB & & & & & $-0.0055^{*}$ & 0.0025 & & & & \\
\hline EWB & & & & & 0.0010 & 0.0026 & & & & \\
\hline Fatigue & & & $0.0083 * * *$ & 0.0015 & $0.0065 * * *$ & 0.0016 & & & $0.0064 * * *$ & 0.0015 \\
\hline TOI & & & & & & & $0.0054 * * *$ & 0.0006 & & \\
\hline ECOG & $-0.2050 * * *$ & 0.0374 & $-0.1944 * * *$ & 0.0372 & $-0.1792 * * *$ & 0.0372 & $-0.1884 * * *$ & 0.0370 & $-0.1870 * * *$ & 0.0368 \\
\hline
\end{tabular}

ECOG is a dummy variable assuming the value of 1 for $E C O G=2$ and 0 otherwise $(E C O G=0.1)$

Model 1 algorithm using FACIT overall score(s), Model 2 algorithm using general (FACT-G) and questionnaire-specific scale(s) separately, Model 3 algorithm using the four FACT-G wellbeing subscales, Model 4 algorithm using FACIT TOI score(s), Model 5 algorithm using TOI subscales separately, GEE generalized estimating equations, $U K$ United Kingdom, $N L$ Netherlands, $S E$ standard error, FACT-G functional assessment of cancer therapy-general, FAACT functional assessment of anorexia/cachexia treatment, FACIT-F functional assessment of chronic illness therapy-fatigue, $P W B$ physical wellbeing, $F W B$ functional wellbeing, $E W B$ emotional wellbeing, $S W B$ social/family wellbeing, ACS Anorexia-Cachexia Subscale, TOI trial outcome index, ECOG Eastern Cooperative Oncology Group

$*^{*}$ value $<0.05$

$* * p$ value $<0.01$

$* * * p$ value $<0.001$

English value set (mappings to the EQ-5D-3L have most commonly used this set) and the Dutch one, which are the two EQ-5D-5L sets of weights closest to the ROMANA trials populations [7].

Second, model validation used a non-random split-sample method, rather than testing the algorithms in an external dataset that would be the preferred approach according to the MAPS Statement [13]. However, significant differences were found in relevant baseline patients characteristics (i.e. $\mathrm{BMI}$, weight loss, ECOG, chemotherapy/radiotherapy status) between the two samples, thus the cross-sample validation was likely to have been conducted on a quasi-different NSCLC-C population. A completely different approach has been suggested by most recent guidelines [33], which recommend not splitting the sample for validation purposes if this implies a further reduction of a (small) sample size.

Third, the mapping functions were developed using a small database, since only 96 patients enrolled in the ROMANA trials were invited to complete the EQ-5D-5L. However, because the patients completed the HRQoL questionnaires on up to five occasions, 420 observations were available for analysis, $79 \%(n=332)$ of which were used to obtain regression coefficients and $21 \%(n=88)$ to validate the mapping algorithms.
Fourth, the sample of NSCLC-C patients who were likely to have a better health status (i.e. ECOG $\leq 2$, life expectancy at least of 4 months) than the general population with the same medical condition due to the ROMANA trials' inclusion criteria. Thus, the generalizability of the developed mapping functions to other NSCLC-C samples should consider any potential clinical differences.

Finally, as already observed in the literature [11,34], all mapping algorithms tend to over-predict utility values for patients in poor health and, conversely, under-predict the highest scores. Moreover, they systematically fail to predict the value of 1 corresponding to perfect health, although in our database, due to the severity of the NSCLC-C condition, less than $20 \%$ of EQ-5D-5L observations were at the ceiling at each study visit, which is lower than observed in another mapping study using the same tool [30]. This bias affected the FACIT-F algorithms less than FACT-G and FAACT ones and, within the same FACIT instrument, models using disaggregated scales (Model 3) instead of summary scores (Model 1). Skaltsa et al. [19] developed groupspecific models according to disease severity to increase prediction accuracy at the "extremes"; unfortunately, due to the small database, this approach was infeasible in this study. As population-average models, the estimated GEE functions 
Table 4 Synthesis of model performance across all visits (validation sample)

\begin{tabular}{|c|c|c|c|c|c|c|}
\hline & Mean $( \pm S D)$ & Range (min, max) & $\%$ floor, $\%$ ceiling & $\begin{array}{l}\text { Mean observed - mean } \\
\text { predicted }\left(p \text { value }^{\mathrm{a}}\right)\end{array}$ & MAE & RMSE \\
\hline \multicolumn{7}{|c|}{ (a) UK weights } \\
\hline Observed & $0.809( \pm 0.16)$ & $0.128,1.000$ & $0.0,14.8$ & - & - & - \\
\hline \multicolumn{7}{|l|}{ FACT-G } \\
\hline Model 1 & $0.816( \pm 0.06)$ & $0.590,0.953$ & $0.0,0.0$ & $-0.0070(0.7017)$ & 0.0997 & 0.1339 \\
\hline Model 3 & $0.832( \pm 0.07)$ & $0.583,0.970$ & $0.0,0.0$ & $-0.0226(0.2335)$ & 0.0942 & 0.1272 \\
\hline \multicolumn{7}{|l|}{ FAACT } \\
\hline Model 1 & $0.827( \pm 0.06)$ & $0.645,0.984$ & $0.0,0.0$ & $-0.0178(0.3393)$ & 0.1027 & 0.1366 \\
\hline Model 2 & $0.825( \pm 0.06)$ & $0.646,0.971$ & $0.0,0.0$ & $-0.0161(0.3857)$ & 0.1064 & 0.1412 \\
\hline Model 3 & $0.835( \pm 0.07)$ & $0.638,0.987$ & $0.0,0.0$ & $-0.0259(0.1722)$ & 0.0995 & 0.1332 \\
\hline Model 4 & $0.819( \pm 0.07)$ & $0.641,0.967$ & $0.0,0.0$ & $-0.0100(0.5927)$ & 0.1027 & 0.1362 \\
\hline Model 5 & $0.831( \pm 0.07)$ & $0.652,0.979$ & $0.0,0.0$ & $-0.0219(0.2455)$ & 0.1015 & 0.1340 \\
\hline \multicolumn{7}{|l|}{ FACIT-F } \\
\hline Model 1 & $0.831( \pm 0.07)$ & $0.544,0.998$ & $0.0,0.0$ & $-0.0223(0.2417)$ & 0.0933 & 0.1267 \\
\hline Model 2 & $0.843( \pm 0.08)$ & $0.575,0.991$ & $0.0,0.0$ & $-0.0343(0.0761)$ & 0.0942 & 0.1288 \\
\hline Model 3 & $0.844( \pm 0.08)$ & $0.577,1.014$ & $0.0,1.1$ & $-0.0351(0.0729)$ & 0.0913 & 0.1261 \\
\hline Model 4 & $0.833( \pm 0.08)$ & $0.543,0.999$ & $0.0,0.0$ & $-0.0241(0.2161)$ & 0.0899 & 0.1230 \\
\hline Model 5 & $0.842( \pm 0.08)$ & $0.553,0.998$ & $0.0,0.0$ & $-0.0328(0.0917)$ & 0.0917 & 0.1257 \\
\hline \multicolumn{7}{|c|}{ (b) NL weights } \\
\hline Observed & $0.760( \pm 0.19)$ & $-0.073,1.000$ & $0.0,14.8$ & - & - & - \\
\hline \multicolumn{7}{|l|}{ FACT-G } \\
\hline Model 1 & $0.766( \pm 0.07)$ & $0.505,0.939$ & $0.0,0.0$ & $-0.0161(0.4561)$ & 0.1094 & 0.1574 \\
\hline Model 3 & $0.784( \pm 0.08)$ & $0.499,0.945$ & $0.0,0.0$ & $-0.0245(0.2719)$ & 0.1068 & 0.1503 \\
\hline \multicolumn{7}{|l|}{ FAACT } \\
\hline Model 1 & $0.778( \pm 0.07)$ & $0.568,0.958$ & $0.0,0.0$ & $-0.0178(0.2991)$ & 0.1131 & 0.1601 \\
\hline Model 2 & $0.780( \pm 0.07)$ & $0.572,0.950$ & $0.0,0.0$ & $-0.0200(0.2586)$ & 0.1174 & 0.1651 \\
\hline Model 3 & $0.788( \pm 0.08)$ & $0.567,0.964$ & $0.0,0.0$ & $-0.0280(0.0932)$ & 0.1112 & 0.1566 \\
\hline Model 4 & $0.776( \pm 0.08)$ & $0.567,0.950$ & $0.0,0.0$ & $-0.0165(0.3365)$ & 0.1145 & 0.1605 \\
\hline Model 5 & $0.787( \pm 0.08)$ & $0.576,0.961$ & $0.0,0.0$ & $-0.0266(0.1118)$ & 0.1126 & 0.1571 \\
\hline \multicolumn{7}{|l|}{ FACIT-F } \\
\hline Model 1 & $0.784( \pm 0.08)$ & $0.453,0.976$ & $0.0,0.0$ & $-0.0238(0.1329)$ & 0.1026 & 0.1481 \\
\hline Model 2 & $0.807( \pm 0.09)$ & $0.488,0.983$ & $0.0,0.0$ & $-0.0471(0.0030)$ & 0.1051 & 0.1515 \\
\hline Model 3 & $0.797( \pm 0.10)$ & $0.492,0.992$ & $0.0,0.0$ & $-0.0370(0.0175)$ & 0.1030 & 0.1471 \\
\hline Model 4 & $0.786( \pm 0.10)$ & $0.453,0.976$ & $0.0,0.0$ & $-0.0262(0.0885)$ & 0.1007 & 0.1440 \\
\hline Model 5 & $0.798( \pm 0.09)$ & $0.464,0.980$ & $0.0,0.0$ & $-0.0383(0.0132)$ & 0.1020 & 0.1464 \\
\hline
\end{tabular}

$U K$ United Kingdom, $N L$ Netherlands, $S D$ standard deviation, FACT-G functional assessment of cancer therapy-general, FAACT functional assessment of anorexia/cachexia treatment, FACIT-F functional assessment of chronic illness therapy-fatigue, MAE mean absolute error, RMSE root mean squared error

${ }^{\mathrm{a}} T$ test

performed well in predicting mean utility values, which are usually required to populate model-based economic evaluations. New techniques such as beta-binomial regression and limited dependent variable mixture (LDVM) models [22, 23, 35], which better fit the typical distributions of EQ-5D data, are emerging in the mapping literature to overcome the wellknown limitations of linear models and might be explored in future research with larger databases.

\section{Conclusion}

Given the increasing costs of cancer care it is important to support the health-related decision-making process of allocating scarce resources by assessing the value of treatments through economic evaluation techniques such as cost-utility analysis [36]. Previous research showed that cancer cachexia, mainly affecting lung cancer patients, has 
a significant impact on patients HRQoL and healthcare resources utilization [10]. This study provided algorithms to predict EQ-5D-5L utility values from FACT-G, FAACT and FACIT-F scores, confirming that mapping may represent a useful tool in the absence of preference-based HRQoL scores. These algorithms could be applied in other studies related to cancer cachexia or NSCLC in general, by those requiring EQ-5D-5L utility values for QALY calculation.

Acknowledgements This study was supported by a grant from Helsinn Therapeutics.

Open Access This article is distributed under the terms of the Creative Commons Attribution 4.0 International License (http://creativecommons.org/licenses/by/4.0/), which permits unrestricted use, distribution, and reproduction in any medium, provided you give appropriate credit to the original author(s) and the source, provide a link to the Creative Commons license, and indicate if changes were made.

\section{References}

1. Evans, W.J., Morley, J.E., Argilés, J., et al.: Cachexia: a new definition. Clin. Nutr. 27, 793-799 (2008)

2. Takayama, K., Atagi, S., Imamura, F., et al.: Quality of life and survival survey of cancer cachexia in advanced non-small cell lung cancer patients-Japan nutrition and QOL survey in patients with advanced non-small cell lung cancer study. Supp. Care Cancer (2016). doi:10.1007/s00520-016-3156-8

3. Le Blanc, T.W., Nipp, R.D., Rushing, C.N., et al.: Correlation between the international consensus definition of the Cancer Anorexia-Cachexia Syndrome (CACS) and patient-centered outcomes in advanced non-small cell lung cancer. J. Pain Symptom Manage. 49(4), 680-689 (2015)

4. Porporato, P.E.: Understanding cachexia as a cancer metabolism syndrome. Oncogenesis 5, e200 (2016). doi:10.1038/ oncsis.2016.3

5. Functional Assessment of Chronic Illness Therapy. http://www. facit.org/. Accessed Apr 2016

6. Blauwhoff-Buskermolen, S., Ruijgrok, C., Ostelo, R.W., et al.: The assessment of anorexia in patients with cancer: cut-off values for the FAACT-A/CS and the VAS for appetite. Supp. Care Cancer 24, 661-666 (2016)

7. European Quality of Life Group Association. http://www.euroqol. org/. Accessed Apr 2016

8. National Institute for Health and Clinical Excellence (NICE): Guide to the methods of technology appraisal. NICE, London (2008)

9. Proskorovsky, I., Lewis, P., Williams, C.D., et al.: Mapping EORTC QLQ-C30 and QLQ-MY20 to EQ-5D in patients with multiple myeloma. DOI, Health Qual Life Outcomes (2014). doi:10.1186/1477-7525-12-35

10. Tarricone, R., Ricca, G., Nyanzi-Wakholi, B., Medina-Lara, A.: Impact of cancer anorexia-cachexia syndrome on health-related quality of life and resource utilization: a systematic review. Crit. Rev. Oncol. Hematol. 99, 49-62 (2016)

11. Teckle, P., McTaggart-Cowan, H., Van der Hoek, K., et al.: Mapping the FACT-G cancer-specific quality of life instrument to the EQ-5D and SF-6D. Health Qual. Life Outcomes (2013). doi:10.1186/1477-7525-11-203
12. Health Economics Research Centre (HERC) database of mapping studies. University of Oxford. http://www.herc.ox.ac.uk/ downloads/herc-database-of-mapping-studies. Accessed Apr 2016

13. Petrou, S., Rivero-Arias, O., Dakin, H., et al.: The MAPS reporting statement for studies mapping onto generic preference-based outcome measures: explanation and elaboration. Pharmacoeconomics 33(10), 993-1011 (2015)

14. Temel, J.S., Abernethy, A.P., Currow, D.C., et al.: Anamorelin in patients with non-small-cell lung cancer and cachexia (ROMANA 1 and ROMANA 2): results from two randomized, double-blind, phase 3 trials. Lancet Oncol. 17, 519-531 (2016)

15. Devlin N, Shah K, Feng Y, Mulhern B, van Hout B.: Valuing health-related quality of life: an EQ-5D-5L value set for England. Office of Health Economics (OHE), Research Paper 16/01, January 2016 (unpublished article)

16. Versteegh, M.M., Vermeulen, K.M., Evers, S.M.A.A., Ardine de Wit, G., Prenger, R., Stolk, E.A.: Dutch tariff for the five-level version of EQ-5D. Value Health 19(4), 343-352 (2016)

17. Ballinger, G.A.: Using generalized estimating equations for longitudinal data analysis. Org. Res. Methods (2004). doi:10.1177/1094428104263672

18. Wang, M.: Generalized estimating equations in longitudinal data analysis: a review and recent developments. Adv. Stat. (2014). doi: $10.1155 / 2014 / 303728$

19. Skaltsa, K., Longworth, L., Ivanescu, C., Holmstrom, S.: Mapping the FACT-P to the preference-based EQ-5D questionnaire in metastatic castration-resistant prostate cancer. Value Health 17, 238-244 (2014)

20. Cui, J.: QIC program and model selection in GEE analyses. Stata J. 7(2), 209-220 (2007)

21. Wu, E.Q., Mulani, P., Farrell, M.H., Sleep, D.: Mapping FACT$P$ and EORTC QLQ-C30 to patient health status measured by EQ-5D in metastatic hormone-refractory prostate cancer patients. Value Health 10(5), 408-414 (2007)

22. Longworth, L., Yang, Y., Young, T., et al.: Use of generic and condition-specific measures of health-related quality of life in NICE decision-making: a systematic review, statistical modelling and survey. Health Technol. Assess. 18(9), 1-224 (2014). doi: $10.3310 /$ hta 18090

23. Khan, I., Morris, S., Pashayan, N., Matata, B., Bashir, Z., Maguirre, J.: Comparing the mapping between EQ-5D-5L, EQ5D-3L and the EORTC-QLQ-C30 in non-small cell lung cancer patients. Health Qual. Life Outcomes (2016). doi:10.1186/ s12955-016-0455-1

24. Starkie, H.J., Briggs, A.H., Chambers, M.G., Jones, P.: Predicting EQ-5D values using the SGRQ. Value Health 14, 354-360 (2011)

25. Arnold, D.T., Rowen, D., Versteegh, M.M., Morley, A., Hooper, C.E., Maskell, N.A.: Testing mapping algorithms of the cancerspecific EORTC QLQ-C30 onto EQ-5D in malignant mesothelioma. Health Qual. Life Outcomes 13, 6 (2015). doi:10.1186/ s12955-014-0196-y

26. Pickard, A.S., Neary, M.P., Cella, D.: Estimation of minimally important differences in EQ-5D utility and VAS scores in cancer. Health Qual. Life Outcomes 5, 70 (2007)

27. Cheung, Y.B., Thumboo, J., Gao, F., et al.: Mapping the English and Chinese versions of the Functional Assessment of Cancer Therapy-General to the EQ-5D utility index. Value Health 12(2), 371-376 (2009)

28. Diels, J., Hamberg, P., Ford, D., et al.: Mapping FACT-P to EQ-5D in a large cross-sectional study of metastatic castrationresistant prostate cancer patients. Qual. Life Res. 24, 591-598 (2015)

29. Askew, R.L., Swartz, R.J., Xing, Y., et al.: Mapping the FACTMelanoma Quality-of-Life Scores to EQ-5D Health Utility Weights. Value Health 14, 900-906 (2011) 
30. Cheung, Y.B., Luo, N., Ng, R., Lee, C.F.: Mapping the functional assessment of cancer therapy-breast (FACT-B) to the 5-level EuroQol Group's 5-dimension questionnaire (EQ-5D-5L) utility index in a multi-ethnic Asian population. Health Qual. Life Outcomes 12, 180 (2014). doi:10.1186/s12955-014-0180-6

31. Versteegh, M.M., Leunis, A., Luime, J.J., Boggild, M., Uyl-de Groot, C.A., Stolk, E.A.: Mapping QLQ-C30, HAQ, and MSIS-29 on EQ-5D. Med. Decis. Mak. 32(4), 554-568 (2012)

32. Kay, S., Tolley, K., Colayco, D., Khalaf, K., Anderson, P., Globe, D.: Mapping EQ-5D utility scores from the Incontinence Quality of Life Questionnaire among patients with neurogenic and idiopathic overactive bladder. Value Health 16, 394-402 (2013)

33. Wailoo, A.J., Hernandez-Alava, M., Manca, A., et al.: Mapping to estimate health-state utility from non-preference-based outcome measures: an ISPOR good practices for outcomes research task force report. Value Health 20(1), 18-27 (2017)

34. Versteegh, M.M., Rowen, D., Brazier, J.A., Stolk, E.A.: Mapping onto Eq-5 D for patients in poor health. Health Qual. Life Outcomes 8, 141 (2010). doi:10.1186/1477-7525-8-141

35. Hernández Alava, M., Wailoo, A.J., Ara, R.: Tails from the peak district: adjusted limited dependent variable mixture models of EQ-5D questionnaire health state utility values. Value Health 15(3), 550-561 (2012)

36. Meropol, N.J., Schrag, D., Smith, T.J., et al.: American Society of Clinical Oncology guidance statement: the cost of cancer care. J. Clin. Oncol. 27(23), 3868-3874 (2009) 